\title{
Investigation of the Interaction between a Bivalent Aptamer and Thrombin by AFM
}

\author{
Lin Ge, ${ }^{,, \neq}$Gang Jin, ${ }^{*,+, \S}$ and Xiaohong Fang ${ }^{*, l l}$ \\ ${ }^{\dagger}$ Institute of Biophysics, Chinese Academy of Sciences, 15 Datun Road, Chaoyang District, 100101 Beijing, China \\ ${ }^{\ddagger}$ Graduate University of the Chinese Academy of Sciences, 19A Yu Quan Road, Shijingshan District, 100049 Beijing, China \\ ${ }^{\S}$ Institute of Mechanics, Chinese Academy of Sciences, 15 Bei-si-huan West Road, 100190 Beijing, China \\ "Institute of Chemistry, Chinese Academy of Sciences, Zhongguancun North First Street 2, 100190 Beijing, China
}

Supporting Information

ABSTRACT: Aptamers are a new class of molecular probes for protein recognition, detection, and inhibition. Multivalent aptamer-protein binding through aptamer assembly has been currently developed as an effective way to achieve higher protein affinity and selectivity. In this study, the specific interaction between bivalent aptamer Bi-8S and thrombin has been measured directly and quantitatively by atomic force microscopy to investigate the unbinding dynamics and dissociation energy landscape of the multivalent interaction. Bivalent aptamer Bi-8S contains thrombin's two aptamers, 15apt and 27apt, which are linked by eight spacer phosphoramidites. The results revealed the sequential dissociation of the two aptamers. Moreover, the dynamic force spectroscopy data


revealed that the 27apt's binding to the thrombin remains largely unaffected by the eight-spacer phosphoramidites within Bi- $8 \mathrm{~S}$. In contrast, the eight-spacer phosphoramidites stabilized the 15 apt-thrombin binding.

\section{INTRODUCTION}

Aptamers are nucleic acid (NA) sequences selected by the systematic evolution of ligands by exponential enrichment (SELEX) method $^{1,2}$ in which single-stranded DNAs or RNAs are isolated from a random sequence library for their ability to bind selectively to a variety of target molecules ranging from ions and small organic or inorganic molecules to proteins and living cells. ${ }^{3-5}$ They specifically recognize targets with binding affinity and selectivity for targets comparable to those of antibodies. ${ }^{6}$ The development and application of aptamers that specifically recognize protein targets and modulate their biological functions through binding with their functional domains have attracted particular attention. ${ }^{7,8}$ They are emerging as a new class of protein probes that rival antibodies in both diagnostic and therapeutic applications. ${ }^{9-13}$ Therefore, the understanding of how aptamers interact with their protein targets is of great theoretical and practical importance.

In comparison with commonly used antibodies, aptamers have many advantages: NA aptamers have much lower molecular weights, so their secondary structures are easily predictable; they are not prone to irreversible denaturation and therefore are easier to store. In addition, they can be synthesized efficiently and reliably by using established phosphoramidite chemistry and are easier to modify. Recently, it has been reported that assembling two thrombin-binding aptamers with optimized design parameters resulted in a bivalent aptamer with a higher thrombin binding affinity. ${ }^{14}$ It demonstrated a new strategy of using aptamer assembly to develop a nucleic acid-based high-performance protein inhibitor through a polyvalent interaction. Polyvalence is ubiquitous in biology, which involves the binding of multiple ligands of a biological entity, such as small molecules, oligosaccharides, proteins, nucleic acids, lipids, and aggregates of these molecules, to multiple binding pockets or receptors of a target (e.g., a protein, virus, bacterium, or cell ${ }^{15}$ ). It has a number of benefits over monovalent interactions, such as possessing a higher binding affinity and providing a higher selectivity in target recognition. ${ }^{16,17}$ By conjugating aptamers together through NA chemistry, rationally designed aptamer assemblies can combine the functionality and binding affinity of different aptamers to achieve greatly enhanced enzymatic inhibition in a simple, effective, and practical way. ${ }^{14}$ However, until now, there has been no investigation of the kinetic parameters and dissociation process of the complex formed by aptamer assembly and protein, which limits the rational design of multivalent aptamers.

Atomic force microscopy offers a convenient way to measure the force that governs specific molecular interactions through the detection of the unbinding events between a receptor-functionalized AFM tip and a ligand-modified substrate. In the past, singlemolecule force spectroscopy (SMFS) was applied successfully to receptor/ligand interactions, ${ }^{19}$ protein/protein interactions, ${ }^{18}$ and the interaction between single strands of $\mathrm{DNA}^{20,33}$ to quantify their molecular interactions. Previous studies of thrombin and its DNA aptamers using AFM have demonstrated great potential for SMFS as an investigation method. ${ }^{6,21,22}$

Received: October 9, 2011

Revised: November 20, 2011

Published: November 21, 2011 
Dynamic force spectroscopy (DFS) probes these interactions at different force loading rates and reveals their kinetic parameters, ${ }^{28,34}$ offering information on the prominent barriers traversed in the energy landscape along their force-driven unbinding pathways. It has been applied to exploring the energy landscape of intermolecular bonds for small-molecule ligand/ protein, ${ }^{29}$ antibody/antigen, ${ }^{30}$ and other ligand/receptor pairs. ${ }^{31}$ Using both techniques, how the interactions, transition state, lifetime, and free-energy difference of the aptamer-thrombin complex change under various conditions can be quantified.

In this article, we present the study of unbinding dynamic force spectra between thrombin and its bivalent aptamer. Bivalent aptamer Bi-8S contains 8 spacer phosphoramidites linking thrombin's two aptamers; one is 15 bases long (15apt) and binds at exosite 1 and the other is 27 bases long (27apt) and interacts with exosite 2 . $^{11,32}$ Thrombin and aptamer are covalently linked to the AFM tips and the silicon surface, respectively. The unbinding forces of the aptamer-thrombin complex were measured under different loading rates and conditions by AFM. We revealed the sequential unbinding of the two aptamers to thrombin. The results showed that the unbinding of both aptamer-thrombin complexes undergoes multiple transition states and overcomes two activation barriers before final separation and that the eight phosphoramidite linkers have different effects on the Bi-8S aptamer's binding to thrombin.

\section{RESULTS AND DISCUSSION}

Strategy of the Direct Single-Molecule Force Measurement of $\boldsymbol{\alpha}$-Thrombin with Its Two Aptamers. The direct measurement of the single-molecule unbinding force of the aptamer-thrombin complex was performed using AFM between a thrombin-modified AFM tip and a DNA aptamermodified silicon substrate. The $\alpha$-thrombin protein was coupled to the AFM tip through a 9- to 10-nm-long poly(ethylene glycol) (PEG) cross-linker. Aptamer Bi-8S containing eight spacer

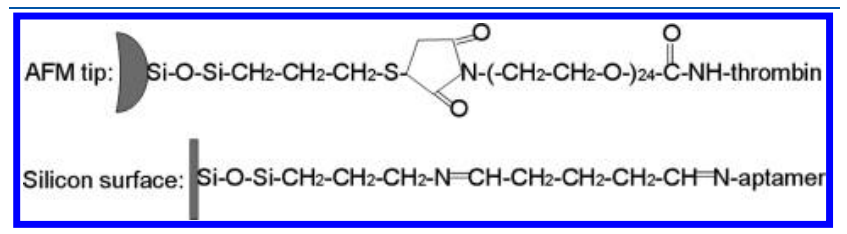

Figure 1. Schematic structures of the $\alpha$-thrombin-functionalized AFM tip and the aptamer sample immobilized on the silicon substrate. phosphoramidites linking thrombin's two aptamers, 15apt and 27apt, is covalently bound to the silicon surface (Figure 1).

The existence of the PEG cross-linker was introduced to allow us to differentiate specific from nonspecific forces on the forcedistance curve. ${ }^{27}$ Within the Bi-8S aptamer, the extra thymine cap at the end of the aptamer (Materials and Methods) allows the aptamer to have sufficient spatial movement freedom. The $8 \mathrm{~S}$ spacer structure is reported to be the optimum length for bivalent aptamer-thrombin complex binding, exhibiting the longest clotting time. ${ }^{14}$ The low density of the immobilized aptamer and thrombin was used to ensure that statistically only one aptamer-thrombin pair could be formed in the contact area between the tip outer apex and the silicon surface; consequently, the force-distance $(F-D)$ curve detected would be from a single aptamer-thrombin complex's interaction.

As shown in Figure 2, we performed direct single-molecule force measurements under various conditions. First, the interactions were probed in buffer solution without any blocking aptamer (Figure 2a) to investigate the sequential unbinding of thrombin's two aptamers from the thrombin. To promote the probability of binding for the aptamers to thrombin, the AFM stylus was pushed toward the silicon surface at $0.5-0.8 \mathrm{nN}$ for about $1 \mathrm{~s}$. Afterward, the stylus was retracted at a certain predetermined speed to record a force-distance curve. Later, the buffer solution was changed to those containing different blocking aptamers: (1) buffer solution containing thrombin's exosite 1-binding 15-base-long aptamer, 15apt, to block the interaction of $\mathrm{Bi}$ $8 \mathrm{~S}$ with thrombin at exosite 1 (Figure 2b), (2) buffer solution containing thrombin's exosite 2-binding 27-base-long aptamer, 27apt, to block the interaction of $\mathrm{Bi}-8 \mathrm{~S}$ with thrombin at exosite 2 (Figure 2c), (3) buffer solution containing both 15apt and 27apt to block Bi-8S's binding (Figure 2d), and (4) a buffer solution containing aptamer Bi-8S, which blocks the anchored Bi-8S's binding (Figure 2e).

The attachment of the aptamer to thrombin could occur at two possible locations: at exosite 1 where 15 apt binds to thrombin or at exosite 2 where 27 apt binds. ${ }^{11,32}$ The binding would remain to be bound until forced to unbind upon an externally applied force by the AFM tip. These ruptures would result in peaks detected in the $F-D$ curves, corresponding to the unbinding of an aptamer-thrombin bond. The process ends after both aptamers have been forced to unbind from their binding sites. The studied system has a structure that contains two cross-linkers connecting the two interacting particles to the AFM tip and the silicon surface. If fully stretched, these two cross-linkers would contribute to



Figure 2. Schematics for the mechanical unbinding of thrombin/aptamer complexes. $\alpha$-Thrombin is covalently bound to the AFM tip via a cross-linker; aptamer Bi-8S is covalently bound to the silicon surface. Single-molecule force spectroscopy was preformed under various conditions including buffer solution (a) without blocking aptamer, (b) with free 15 aptamer (blue square) blocking, (c) with free 27 aptamer (red triangle) blocking, (d) with both free 15 and 27 aptamer blocking, and (e) with free Bi-8S aptamer blocking. 

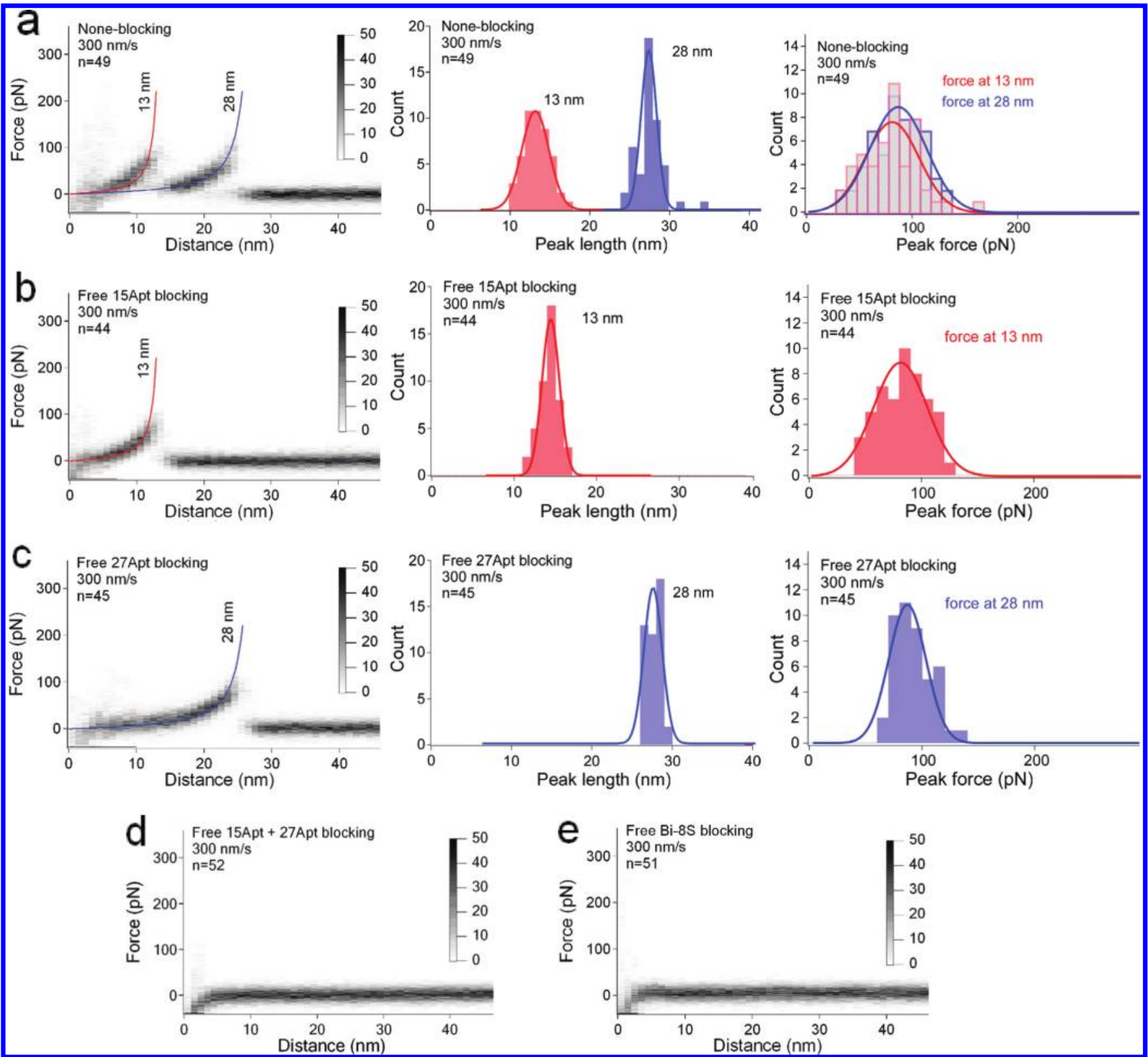

Figure 3. Force spectroscopy of thrombin-aptamer unbinding. Superimposition of force-distance $(F-D)$ curves recorded at a pulling speed of $300 \mathrm{~nm} / \mathrm{s}$ in buffer solution (a) without blocking aptamer, (b) with free 15 aptamer blocking, (c) with free 27 aptamer blocking, (d) with both free 15 and 27 aptamer blocking, and (e) with free Bi-8S aptamer blocking. The buffer solution used was $140 \mathrm{mM} \mathrm{NaCl}, 5 \mathrm{mM} \mathrm{KCl}, 1 \mathrm{mM} \mathrm{CaCl}_{2}, 1 \mathrm{mM} \mathrm{MgCl}$, and $20 \mathrm{mM}$ Tris- $\mathrm{HCl}$ at $\mathrm{pH}$ 7.4. The concentration of free aptamer for blocking is $1.0 \times 10^{-7} \mathrm{M}$ in buffer. Every reproducibly occurring force peak was then fitted using the WLC model (Materials and Methods) to determine the rupture force as well as the contour length of the thrombin-aptamer binding. The contour length is labeled at the end of each WLC fit, approximately at $13 \mathrm{~nm}$ (red) and $28 \mathrm{~nm}$ (blue). The images on the right under conditions $a-c$ show histograms mapping the distribution of the peak contour length and the rupture force. $n$ indicates the number of curves superimposed in each image, with the color bar shown on the right.

a combined contour length of 11-13 nm. Meanwhile, within aptamer Bi-8S there exist eight spacer phosphoramidites; if these spacers are fully stretched, then the expected length interval should lie within 16 to $17 \mathrm{~nm}$. Consider also that the thrombin molecule has a typical diameter of 2 to $3 \mathrm{~nm}$, making the total contour length 29-33 nm. Because of our immobilization method with a low concentration of substrates, we caused the disadvantage of reducing the binding probability, resulting in more force curves having no rupture events. However, this disadvantage is compensated for by increasing the amount of the force-distance cycle recorded until eventually a sufficient number of force curves were acquired for superimposition under each condition and loading rate, as shown in Figure S1. Force curves with no rupture peaks were not included in the data analysis.

All force curves were then superimposed to weaken the effect of occasional nonspecific interaction at the beginning of the curve while promoting their dominant features (Figure S1, Figure 3). The superimposition showed sawtooth-like patterns with each force peak characterizing the corresponding unbinding of one aptamer-thrombin bond. Fitting every force peak of every $F-D$ curve with the 

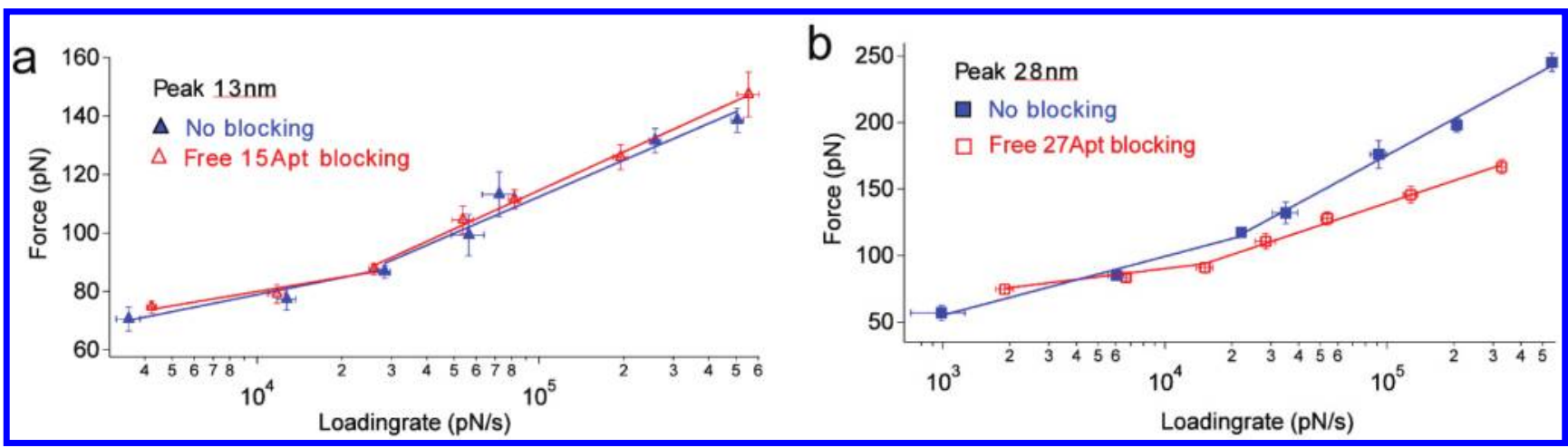

Figure 4. DFS spectra recorded upon aptamer-thrombin unbinding, setting subjects in buffer solution in the absence (solid blue) and presence (hollow red) of free aptamers. The most probable forces and SE (error bars) were determined from the Gaussian fits for all reproducibly occurring force peaks (interactions) shown in Figure S1. (a) Spectra of the interaction between 27apt and thrombin at the 27apt binding site (exosite 2, contour length $13 \mathrm{~nm}$ ). Blue data points represent the data of force curves from the buffer solution without free aptamers. Red data points represent the data of force curves from the buffer solution with free 15apt blocking. (b) Spectra of the interaction between 15apt and thrombin at the 15apt binding site (exosite 1, contour length $28 \mathrm{~nm}$ ). Blue data points represent the data of the force curves from buffer solution without free aptamers. Red data points represent the data of force curves from buffer solution with free 27apt blocking.

worm-like-chain (WLC) model (red and blue curves in Figure 3) revealed average contour lengths of the stretched polymer. ${ }^{35,36}$

SMFS Reveals the Interaction Pattern of the Thrombin-Aptamer Bond. When the thrombin and Bi-8S aptamer were set in buffer solution without blocking aptamers (Figure 3a), two pronounced force peaks were clearly visible in the superimposition at the most probable contour lengths of 13 and $28 \mathrm{~nm}$, which is in agreement with the expected contour length. Measuring the magnitude of each force peak in every force curve provided the strengths of the interactions that have been established. The histogram mapping of the distribution of the rupture contour length and the rupture force is shown to the right of the superimposition. The pulling speed in the presented data was $300 \mathrm{~nm} / \mathrm{s}$. $n$ indicates the number of force curves superimposed.

Upon exposure to excessive free blocking 15apt in the buffer solution (Figure $3 \mathrm{~b}$ ), only one force peak was visible at the most probable contour length of $13 \mathrm{~nm}$, and the other force peak previously seen at a contour length of $28 \mathrm{~nm}$ vanished. This phenomenon falls in line with the fact that when exosite 1 is occupied by the excessive free 15apt in solution, the 15apt component within the $\mathrm{Bi}-8 \mathrm{~S}$ aptamer could no longer bind to the thrombin. Therefore, the remaining force peak in the superimposition was the result of the unbinding of 27apt within the Bi-8S aptamer bound at exosite 2 to the thrombin. During this process, the unbound 15apt component was still linked by the eight-spacer phosphoramidites to the bound 27apt component; therefore, the Bi-8S aptamer most likely will form an alternate conformation other than that under the previous condition. The histogram mapping of the distribution of the rupture contour length and the rupture force is shown to the right of the superimposition.

When excessive free blocking 27apt was introduced into the buffer solution (Figure 3c), only one force peak was visible at the most probable contour length of $28 \mathrm{~nm}$. When exosite 2 is occupied by excess free $27 \mathrm{apt}$, the 27 apt component within the $\mathrm{Bi}-8 \mathrm{~S}$ aptamer could no longer bind to the thrombin. Therefore, the remaining force peak in the superimposition was the result of the unbinding of 15 apt within the $\mathrm{Bi}-8 \mathrm{~S}$ aptamer bound at exosite 1 to the thrombin. During this process, the unbound 27apt component was still linked by the eight-spacer phosphoramidites to the bound 15apt component; therefore, the Bi-8S aptamer most likely will form another alternate conformation other than that under the previous conditions. The histogram mapping of the distribution of the rupture contour length and the rupture force is shown to the right of the superimposition.

Finally, adding excess free 15apt and 27apt (Figure 3d) or free $\mathrm{Bi}-8 \mathrm{~S}$ aptamer to the buffer solution (Figure $3 \mathrm{e}$ ) would take over the binding at exosite 1 and exosite 2, hence blocking the interactions between thrombin and the anchored $\mathrm{Bi}-8 \mathrm{~S}$ aptamer, consequently showing no obvious force peak in the $F-D$ curves.

Under each condition, the WLC fits used the same persistence length (Data Analysis), and the rupture contour length and rupture force histograms both showed a single Gaussian distribution. Both phenomena further demonstrated that the acquired $F-D$ curves were from a single aptamer-thrombin complex. Therefore, it can be concluded that the force peaks shown in Figure 3a were the results of the sequential unbinding of the aptamer-thrombin complex. In comparison with the control experiments in Figure $3 b-e$, we can now assign the rupture force peak at $13 \mathrm{~nm}$ to the unbinding of the 27apt component within $\mathrm{Bi}-8 \mathrm{~S}$ to thrombin at exosite 2 and the rupture force peak at $28 \mathrm{~nm}$ to the unbinding of the 15 apt component within the $\mathrm{Bi}-8 \mathrm{~S}$ aptamer to thrombin at exosite 1. During the process, the 15apt and 27apt components were linked by the eight-spacer phosphoramidites.

As shown in Figure $3 \mathrm{a}-\mathrm{c}$ at a pulling speed of $300 \mathrm{~nm} / \mathrm{s}$, the most probable single-molecule rupture force of thrombinaptamer was about $95 \mathrm{pN}$. This value was comparable to those in our previous study and in other studies. ${ }^{6,22,23}$ It is noted that Basnar et al. ${ }^{21}$ reported the rupture force for a single aptamerthrombin complex to be $\sim 4.45 \mathrm{pN}$ using an aptamer-modified AFM tip to probe the thrombin-modified gold surface and proposed that this rupture force corresponds to the melting of $G$ quadruplexes. However, their result was significantly lower than in other studies of interaction between a DNA fragment and its binding protein. ${ }^{6,8,22-26}$ The reason for this difference might be that the dense coverage of aptamer and thrombin on both surfaces in their system did not provide the ideal binding circumstance. Using an inverted system ${ }^{6,8}$ and an optimized modification method, ${ }^{18}$ we arranged a system with a low concentration of samples to probe the DNA aptamer-functionalized surface, with thrombin tethered on the AFM tip. The system resulted in the generation of a single-molecule interaction, enabling us to 
Table 1. Parameters Characterizing the Energy Barriers $\left(x_{\mathrm{u}}, k_{0}\right.$, and $\left.\Delta G_{\mathrm{u}}\right)$ of Aptamer-Thrombin Binding ${ }^{a}$

\begin{tabular}{|c|c|c|c|c|c|c|}
\hline \multirow[b]{3}{*}{ peak position } & \multicolumn{6}{|c|}{$x_{\mathrm{u}}(\mathrm{nm})$} \\
\hline & \multicolumn{2}{|c|}{ none blocking } & \multicolumn{2}{|c|}{ free 15 apt blocking } & \multicolumn{2}{|c|}{ free 27 apt blocking } \\
\hline & outer & inner & outer & inner & outer & inner \\
\hline $13 \mathrm{~nm}$ & $0.55 \pm 0.13$ & $0.23 \pm 0.03$ & $0.59 \pm 0.15$ & $0.22 \pm 0.01$ & & \\
\hline \multirow{3}{*}{\multicolumn{7}{|c|}{$\begin{array}{l}27 \text { apt @ exosite2 } \\
28 \mathrm{~nm} \\
15 \text { apt @ exosite1 }\end{array}$}} \\
\hline & & & & & & \\
\hline & & & & & & \\
\hline \multirow[b]{3}{*}{ peak position } & \multicolumn{6}{|c|}{$k_{0}\left(\mathrm{~s}^{-1}\right)$} \\
\hline & \multicolumn{2}{|c|}{ none blocking } & \multicolumn{2}{|c|}{ free 15 apt blocking } & \multicolumn{2}{|c|}{ free 27 apt blocking } \\
\hline & outer & inner & outer & inner & outer & inner \\
\hline $13 \mathrm{~nm}$ & $0.04 \pm 0.09$ & $10.99 \pm 6.96$ & $0.016 \pm 0.04$ & $12.79 \pm 2.24$ & & \\
\hline \multicolumn{7}{|l|}{ 27apt @ exosite2 } \\
\hline $28 \mathrm{~nm}$ & $2.93 \pm 1.51$ & $29.3 \pm 4.71$ & & & $0.016 \pm 0.01$ & $12.81 \pm 2.77$ \\
\hline \multicolumn{7}{|l|}{ 15apt @ exosite1 } \\
\hline & \multicolumn{6}{|c|}{$\Delta G\left(k_{\mathrm{B}} \mathrm{T}\right)$} \\
\hline & \multicolumn{2}{|c|}{ none blocking } & \multicolumn{2}{|c|}{ free 15 apt blocking } & \multicolumn{2}{|c|}{ free 27 apt blocking } \\
\hline peak position & outer & inner & outer & inner & outer & inner \\
\hline $13 \mathrm{~nm}$ & $23.9 \pm 2.2$ & $18.3 \pm 0.6$ & $24.9 \pm 2.7$ & $18.2 \pm 0.2$ & & \\
\hline \multicolumn{7}{|l|}{ 27apt @ exosite2 } \\
\hline $28 \mathrm{~nm}$ & $19.7 \pm 0.5$ & $17.4 \pm 0.2$ & & & $24.8 \pm 0.7$ & $18.2 \pm 0.2$ \\
\hline \multicolumn{7}{|l|}{ 15apt @ exosite1 } \\
\hline \multicolumn{7}{|c|}{$\begin{array}{l}{ }^{a} \text { Average values are shown for the energy barriers that stabilize each aptamer's binding with thrombin. Changes in the transition state, } x_{\mathrm{w}} \text { and barrier } \\
\text { heights, } \Delta G_{\mathrm{u}} \text {, were considered to be significant when the values did not overlap within their standard deviations. Changes in the transition rate, } k_{0} \text {, were } \\
\text { considered to be significant when exceeding one order of magnitude. Values that showed a significant difference are highlighted in bold. Errors in } x_{\mathrm{u}} \text { and } \\
k_{0} \text { represent the standard deviation. Errors in } \Delta G_{\mathrm{u}} \text { were estimated by propagating the errors in } k_{0} \text { (Materials and Methods). }\end{array}$} \\
\hline
\end{tabular}

assign the interaction with each aptamer and characterize their interactions.

Dynamic Force Spectrum Reveals the Energetic and Kinetic Properties of the Thrombin-Aptamer Bond. With single-molecule force spectroscopy (SMFS) experiments we studied the interactions established by thrombin with the Bi-8S aptamer and identified the interaction for each component. The interaction strength stabilizing a structure against an externally applied force depends on the force-loading rate applied to overcome the binding energy barrier. ${ }^{35,37}$ Quantifying the average interaction strength for a wide variety of loading rates allows us to estimate the width and height of the free unfolding energy barriers and how they determine the kinetic and mechanical properties of the structural region. ${ }^{38,39}$ To investigate further the effect of each $\mathrm{Bi}-8 \mathrm{~S}^{\prime}$ component on the kinetic and mechanical properties of thrombin-aptamer binding, we analyzed the dynamic force spectrum (DFS) at pulling speeds of 100, 300, 600, 900, 1200, 2400 , and $5000 \mathrm{~nm} / \mathrm{s}$ in the buffer solutions discussed previously (Figure S1) and located the most prominent energy barriers along the reaction coordinate.

From these DFS data, we determined the most probable rupture forces of each unbinding between thrombin and its aptamers. The DFS spectra (Figure 4) of the two thrombin-aptamer unbinding events could be approximated by two linear regimes. According to the Bell-Evans model, ${ }^{40}$ the existence of two linear regimes indicates that two energy barriers separate the native from the unbound state. At low loading rates, the outer barrier was probed, and at high loading rates, the inner barrier governs the unbinding rate.

These energy barriers determined from the DFS data showed inner barriers' ground-transition-state distances, $x_{\mathrm{u}}$, from 0.11 to $0.22 \mathrm{~nm}$ and unfolding rate, $k_{0}$, from 11 to $29 \mathrm{~s}^{-1}$. In contrast, their outer barriers exhibited much larger distances to the transition states, ranging from 0.22 to $0.59 \mathrm{~nm}$, and much lower unfolding rates from 0.016 to $2.9 \mathrm{~s}^{-1}$ (Table 1 ).

For interactions at a contour length of $13 \mathrm{~nm}$ (Figure 4a), where 27apt detaches from thrombin at exosite 2 , the $x_{\mathrm{u}}, k_{0}$, and $\Delta G_{\mathrm{u}}$ values for the barriers under the two conditions (with or without 15apt blocking) showed no significant differences upon exposure to excess free $15 \mathrm{apt}$. This indicates that 27apt's binding to thrombin at exosite 2 remains largely unaffected by the eightspacer phosphoramidites within the Bi-8S.

However, significant differences were detected for interactions at a contour length of $28 \mathrm{~nm}$ (Figure 4b), where 15apt detaches from thrombin at exosite 1 . In the presence of excess free 27apt blocking, $x_{\mathrm{u}}$ for both barriers increased significantly by a factor of up to 2.5. An increase in $x_{\mathrm{u}}$ signifies that the energy valley stabilizing a structural segment becomes wider. Consequently, these structures can adopt more conformational substates. The unbinding rates, $k_{0}$, decreased significantly by up to 2 orders of magnitude, thereby increasing the lifetime. The free unfolding 
energies, $\Delta G_{\mathrm{u}}$, ranging from $17 k_{\mathrm{B}} T$ to $25 k_{\mathrm{B}} T$, also changed for this interaction; in the presence of excess free 27apt, $\Delta G_{\mathrm{u}}$ increased by up to $20 \%$. These changes indicate that the eight-spacer phosphoramidites within $\mathrm{Bi}-8 \mathrm{~S}$ play a role in the 15 apt's binding to thrombin at exosite 1 .

The Bi-8S aptamer is specifically designed to bind to thrombin with high affinity and modulate its function. ${ }^{14}$ However, the underlying molecular mechanisms of such binding are not yet clear. Here we demonstrated that SMFS is capable of quantifying molecular interactions established between thrombin and its novel bivalent aptamer $\mathrm{Bi}-8 \mathrm{~S}$. In addition, the energy properties of these interactions were characterized by probing their dynamic response by DFS. The DFS spectra of thrombin-aptamer binding could be approximated by two linear regimes. From the parameters acquired from such spectra, the experiments showed in detail how the aptamer-thrombin complexes behave energetically and kinetically with respect to an externally applied force during unbinding. It is interesting to find that the existence of the $8 \mathrm{~S}$ linker changed the binding properties of 15apt to thrombin. The linker probably reduced the conformational state and thereby the flexibility of this binding while enhancing the lifetime of this state by up to 2 orders of magnitude.

\section{CONCLUSIONS}

We have quantitatively measured the individual unbinding forces of the Bi- $8 \mathrm{~S}$-thrombin complex upon different loading rates under different circumstances with AFM. The bivalent Bi$8 \mathrm{~S}$ aptamer is assembled from two thrombin-binding aptamers (15apt and 27apt) linked by an eight-phosphoramidite linker. From the SMFS results we observed the sequential unbinding of $\mathrm{Bi}-8 \mathrm{~S}$ and thrombin and assigned the interaction with each aptamer component. With the DFS experiment, we conclude that during the unbinding of both 15apt and 27apt to thrombin one intermediate state with two activation barriers to overcome is encountered. We propose that 27apt's binding to thrombin at exosite 2 remains largely unaffected by the eight-spacer phosphoramidites within the $\mathrm{Bi}-8 \mathrm{~S}$. In the contrast, the eight-spacer phosphoramidite linker plays a role in the 15apt's binding to thrombin at exosite 1 . The conformation formed by the eightphosphoramidites linker stabilized the 15apt-thrombin binding.

\section{MATERIALS AND METHODS}

Materials. Human $\alpha$-thrombin was purchased from Haematologic Technologies Inc. (Essex Junction, VT, U.S.A.). $\alpha$-Thrombin DNA aptamer 1 (15 apt: $5^{\prime}$-GGTTG GTGTG GTTGG-3'), $\alpha$-thrombin DNA aptamer 2 (27 apt: $5^{\prime}$-GTCCG TGGTA GGGCA GGTTG GGGTG AC-3 ${ }^{\prime}$ ), and amine-modified bivalent $\alpha$-thrombin DNA aptamer 3 (Bi-8S: $5^{\prime}-\mathrm{NH}_{2}$-TTTT GTCCG TGGTA GGGCA GGTTG GGGTG ACT-SSSS SSSS-TGGTT GGTGT GGTTG G-3', S indicates one unit of the phosphoramidite spacer) were all synthesized from Biomers GmbH (Ulm, Germany). NHS-PEG 24 -MAL (succinimidyl[( $N$-maleimidopropionamido)-tetracosaethleneglycol] ester, MW 1400) was purchased from Thermo Scientific (Rockford, IL, U.S.A.). (3-Mercaptopropyl) trimethoxysilane (MPTMS), 3-aminopropyltriethoxysilane (APTES), and toluene (99.99\%, HPLC grade) were purchased from Sigma (U.S. A.). Other reagents used in all experiments were of analytical grade. Milli-Q-purified water $(18.2 \mathrm{M} \Omega \cdot \mathrm{cm})$ was used for all experiments.

Preparation and Modification of Silicon Substrates and AFM Tips. According to the previously reported procedures, ${ }^{6}$ a singlecrystal silicon wafer polished on one side was cut into pieces of approximately $1.5 \mathrm{~cm} \times 1.5 \mathrm{~cm}$ before cleaning and modification.
The wafers were cleaned and oxidized by heating to $90{ }^{\circ} \mathrm{C}$ in piranha solution $\left(7: 3 \mathrm{v} / \mathrm{v} 98 \% \mathrm{H}_{2} \mathrm{SO}_{4} / \mathrm{H}_{2} \mathrm{O}_{2}\right)$ for $30 \mathrm{~min}$. Then the cleaned wafers were immediately transferred to a $1.0 \% \mathrm{v} / \mathrm{v}$ APTES in toluene solution and incubated for $2 \mathrm{~h}$; afterward, the unbound silanes were washed away by extensive rinsing with toluene. The silanized wafers were activated by incubation in a solution of glutaraldehyde $(0.1 \% \mathrm{v} / \mathrm{v})$ in Tris- $\mathrm{HCl}$ buffer $(20 \mathrm{mM}$ Tris- $\mathrm{HCl}$ buffer, $140 \mathrm{mM} \mathrm{NaCl}, 5 \mathrm{mM} \mathrm{KCl}$, $1 \mathrm{mM} \mathrm{CaCl}_{2}, 1 \mathrm{mM} \mathrm{MgCl}_{2}, \mathrm{pH}$ 7.4) for $30 \mathrm{~min}$ at room temperature and then rinsed with buffer. The activated wafers were immersed in a solution of aptamer $\left(1.0 \times 10^{-7} \mathrm{M}\right.$ in buffer $)$ at $4{ }^{\circ} \mathrm{C}$ for $10 \mathrm{~h}$. After they were rinsed with buffer, the functionalized substrates were stored in the buffer at $4{ }^{\circ} \mathrm{C}$ until use.

$\mathrm{Si}_{3} \mathrm{~N}_{4}$ AFM cantilevers that were $70 \mu \mathrm{m}$ long (NPS series, Veeco, U.S. A.) were transferred to a solution of MPTMS $(1.0 \% \mathrm{v} / \mathrm{v})$ in toluene, incubated for $2 \mathrm{~h}$ at room temperature, and then rinsed thoroughly with toluene to remove any unbound silane. The silanized tips were then activated by incubation in NHS-PEG ${ }_{24}-\mathrm{MAL}(1 \mathrm{mg} / \mathrm{mL})$ in dimethyl sulfoxide for $3 \mathrm{~h}$ at room temperature and then rinsed extensively with dimethyl sulfoxide to remove any unbound NHS-PEG $24-\mathrm{MAL}$. The activated tips were immersed in a solution of protein $(2 \mathrm{mg} / \mathrm{mL}$ $\alpha$-thrombin in Tris- $\mathrm{HCl}$ buffer) and incubated at room temperature for $30 \mathrm{~min}$. After being rinsed with buffer, the functionalized tips were stored in buffer at $4{ }^{\circ} \mathrm{C}$ until use. This optimized immobilization method ${ }^{18}$ adjusts the density of thrombin on the tip to best meet the expectation that only one thrombin may interact with surface-bound aptamers. More specifically, the AFM tip carries many thrombin molecules; however, their surface density was chosen to be sufficiently low so that on average only about one of the flexibly linked thrombins is expected to be bound to the tip apex end, which will reach aptamer molecules on the surface.

SMFS and DFS. Force measurements were performed with thrombin-functionalized tips and aptamer-modified silicon wafers via AFM (Nanowizard II, JPK Instruments, Germany). Spring constants of individual cantilevers were calibrated in solution using the thermal noise technique. ${ }^{41}$ Functionalized cantilevers have nominal spring constants of $\sim 90 \mathrm{pN} / \mathrm{nm}$ at resonance frequencies of 4.8 to $5.5 \mathrm{kHz}$ in buffer solution. The experimental buffer solution used was Tris-HCl buffer (20 mM Tris- $\mathrm{HCl}, 140 \mathrm{mM} \mathrm{NaCl}, 5 \mathrm{mM} \mathrm{KCl}, 1 \mathrm{mM} \mathrm{CaCl}_{2}, 1 \mathrm{mM}$ $\mathrm{MgCl}_{2}, \mathrm{pH}$ 7.4). The buffer solution was freshly made using nanopure water $(18.2 \mathrm{M} \Omega \cdot \mathrm{cm})$ and p.a. purity grade reagents from Sigma. After the aptamer-modified silicon wafer was immersed into the experimental buffer, the AFM setup was thermally equilibrated for $\sim 30 \mathrm{~min}$. The AFM stylus was pushed onto the substrate at a force of $\sim 0.5-0.8 \mathrm{nN}$ for $1 \mathrm{~s}$. This promoted the specific binding of the aptamer to $\alpha$-thrombin. Then the stylus was withdrawn from the surface at a given pulling velocity. DFS experiments were performed at seven pulling velocities $(100,300,600,900,1200,2400$, and $5000 \mathrm{~nm} / \mathrm{s})$. Under each loading rate for each condition, at least three AFM cantilevers were used.

Data Analysis. Igor Pro software (Wavemetrics Inc., U.S.A.) and home-written macros were used for data analysis. All force peaks of superimposed $F-D$ curves were fitted by the WLC model ${ }^{42}$ with a persistence length of $0.4 \mathrm{~nm} .{ }^{35,42}$ The experimental force loading rate and force histograms were fitted with Gaussian distributions. The relationship between the most probable unbinding force $F^{*}$ and the most probable loading rate $r_{\mathrm{f}}^{*}$ can be described by ${ }^{40,43}$

$$
F^{*}=\left(\frac{k_{\mathrm{B}} T}{x_{\mathrm{u}}}\right) \ln \left(\frac{x_{\mathrm{u}} r_{\mathrm{f}} *}{k_{\mathrm{B}} T k_{0}}\right)
$$

where $k_{\mathrm{B}}$ is the Boltzmann constant, $T$ is the absolute temperature, $x_{\mathrm{u}}$ is the distance between free-energy minimum and transition-state barrier, and $k_{0}$ is the unfolding rate at zero force. The force loading rate was calculated using the slope of the force peak times the pulling velocity. The resulting $F^{*}$ was semilogarithmically plotted versus $r_{\mathrm{f}}^{*} . x_{\mathrm{u}}$ and $k_{0}$ were obtained by fitting the above equation using a nonlinear least-squares algorithm. 
The height of the free-energy barrier, $\Delta G_{\mathrm{w}}$ which separates the folded and unfolded states, was assessed using an Arrhenius equation, ${ }^{44}$

$$
\Delta G_{\mathrm{u}}=-k_{\mathrm{B}} T \ln \left(\tau_{\mathrm{D}} k_{0}\right)
$$

where $\tau_{\mathrm{D}}$ denotes the diffusive relaxation time. Values of $\tau_{\mathrm{D}}$ found for proteins are on the order of $10^{-7}-10^{-9} \mathrm{s.}^{44,45}$ We used $\tau_{\mathrm{D}}=10^{-9} \mathrm{~s}$ throughout all of our calculations, which has also been used for molecular dynamics simulations of protein unfolding. ${ }^{46,47}$ Errors in $\Delta G_{\mathrm{u}}$ were estimated by the propagation of the errors of $k_{0}$.

\section{ASSOCIATED CONTENT}

S Supporting Information. Superimposition of $F-D$ curves used for analysis. This material is available free of charge via the Internet at http://pubs.acs.org.

\section{AUTHOR INFORMATION}

\section{Corresponding Author}

*E-mail: xfang@iccas.ac.cn and gajin@imech.ac.cn.

\section{ACKNOWLEDGMENT}

We thank Le Mu for experimental advice and Mehdi Damaghi and Prof. Daniel Müller for helpful discussions. This work was supported by the Chinese Academy of Sciences and the National Basic Research Program of China (2011CB911001) and the NSFC (no. 20821003).

\section{ABBREVIATIONS:}

aa, amino acids; AFM, atomic force microscopy; DFS, dynamic force spectroscopy; $F-D$, force-distance; WLC, wormlike chain; NAs, nucleic acids.

\section{REFERENCES}

(1) Tuerk, C.; Gold, L. Science 1990, 249, 505.

(2) Ellington, A. D.; Szostak, J. W. Nature 1990, 346, 818.

(3) Jenison, R. D.; Gill, S. C.; Pardi, A.; Polisky, B. Science 1994, 263, 1425 .

(4) Jellinek, D.; Lynott, C. K.; Rifkin, D. B.; Janjić, N. Proc. Natl. Acad. Sci. U.S.A. 1993, 90, 11227.

(5) Wiegand, T. W.; Williams, P. B.; Dreskin, S. C.; Jouvin, M. H.; Kinet, J. P.; Tasset, D. I. Immunol. 1996, 157, 221.

(6) Yu, J.; Jiang, Y.; Ma, X.; Lin, Y.; Fang, X. Chem.-Asian I. 2007, 2, 284.

(7) Fang, X.; Tan, W. Acc. Chem. Res. 2010, 43, 48.

(8) Liu, X.; Jiang, Z.; Zhang, Y.; Wang, Z.; Feng, F.; Wang, S.; Zhang, X. Langmuir 2007, 23, 9140.

(9) Bock, L. C.; Griffin, L. C.; Latham, J. A.; Vermaas, E. H.; Toole, J. I. Nature 1992, 355, 564.

(10) DeAnda, A., Jr.; Coutre, S. E.; Moon, M. R.; Vial, C. M.; Griffin, L. C.; Law, V. S.; Komeda, M.; Leung, L. L.; Miller, D. C. Ann. Thorac. Surg. 1994, 58, 344.

(11) Griffin, L. C.; Tidmarsh, G. F.; Bock, L. C.; Toole, J. J.; Leung, L. L. Blood 1993, 81, 3271.

(12) Ruckman, J.; Green, L. S.; Beeson, J.; Waugh, S.; Gillette, W. L.; Henninger, D. D.; Claesson-Welsh, L.; Janjić, N. I. Biol. Chem. 1998, $273,20556$.

(13) Huang, J.; Moore, J.; Soffer, S.; Kim, E.; Rowe, D.; Manley, C. A.; O’Toole, K.; Middlesworth, W.; Stolar, C.; Yamashiro, D.; Kandel, J. I. Pediatr. Surg. 2001, 36, 357.

(14) Kim, Y.; Cao, Z.; Tan, W. Proc. Natl. Acad. Sci. U.S.A. 2008, 105, 5664 .
(15) Mammen, M.; Choi, S. K.; Whitesides, G. M. Angew. Chem.. Int. Ed. 1998, 37, 2755.

(16) Yuan, C. L.; Reiko, T. L. Acc. Chem. Res. 1995, 28, 321.

(17) Chen, H.; Privalsky, M. L. Proc. Natl. Acad. Sci. U.S.A. 1995, $92,422$.

(18) Hinterdorfer, P.; Baumgartner, W.; Gruber, H. J.; Schilcher, K.; Schindler, H. Proc. Natl. Acad. Sci. U.S.A. 1996, 93, 3477.

(19) Moy, V. T.; Florin, E. L.; Gaub, H. E. Science 1994, 266, 257.

(20) Lee, G. U.; Chrisey, L. A.; Colton, R. I. Science 1994, 266, 771.

(21) Basnar, B.; Elnathan, R.; Willner, I. Anal. Chem. 2006, 78, 3638.

(22) Miyachi, Y.; Shimizu, N.; Ogino, C.; Kondo, A. Nucleic Acids Res. 2010, 38, e21.

(23) Eckel, R.; Wilking, S. D.; Becker, A.; Sewald, N.; Ros, R; Anselmetti, D. Angew. Chem. Int. Ed. 2005, 44, 3921.

(24) Baumgarth, B.; Bartels, F. W.; Becker, A.; Anselmetti, D.; Ros, R. Microbiology 2005, 151, 259.

(25) Bartels, F. W.; Baumgarth, B.; Anselmetti, D.; Ros, R.; Becker, A. I. Struct. Biol. 2003, 143, 145.

(26) Strunz, T.; Oroszlan, K.; Schaefer, R.; Guentherodt, H. J. Proc. Natl. Acad. Sci. U.S.A. 1999, 96, 11277.

(27) Kienberger, F.; Ebner, A.; Gruber, H.; Hinterdorfer, P. Acc. Chem. Res. 2006, 39, 29.

(28) Kedrov, A.; Appel, M.; Baumann, H.; Ziegler, C.; Müller, D. J. I. Mol. Biol. 2008, 375, 1258.

(29) Merkel, R.; Nassoy, P.; Leung, A.; Ritchie, K.; Evans, E. Nature 1999, 397, 50.

(30) Schwesinger, F.; Ros, R.; Strunz, T.; Anselmetti, D.; Güntherodt, H. J.; Honegger, A.; Jermutus, L.; Tiefenauer, L.; Pluckthun, A. Proc. Natl. Acad. Sci. U.S.A. 2000, 97, 9972.

(31) Bustanji, Y.; Arciola, C. R.; Conti, M.; Mandello, E.; Montanaro, L.; Samorí, B. Proc. Natl. Acad. Sci. U.S.A. 2003, 100, 13292.

(32) Tasset, D. M.; Kubik, M. F.; Steiner, W. L. Mol. Biol. 1997, $272,688$.

(33) Yu, J.; Sun, S.; Jiang, Y.; Ma, X.; Chen, F.; Zhang, G.; Fang, X. Polymer 2006, 47, 2533.

(34) Evans, E. Annu. Rev. Biophvs. Biomol. Struct. 2001, 30, 105.

(35) Oesterhelt, F.; Oesterhelt, D.; Pfeiffer, M.; Engel, A.; Gaub, H. E.; Müller, D. I. Science 2000, 288, 143.

(36) Kedrov, A.; Janovjak, H.; Sapra, K. T.; Müller, D. I. Annu. Rev. Biophvs. Biomol. Struct. 2007, 36, 233.

(37) Bell, G. I. Science 1978, 200, 618.

(38) Janovjak, H.; Sapra, K. T.; Kedrov, A.; Müller, D. I. ChemPhusChem 2008, 9, 954.

(39) Bippes, C. A.; Zeltina, A.; Casagrande, F.; Ratera, M.; Palacin, M.; Müller, D. J.; Fotiadis, D. I. Biol. Chem. 2009, 284, 18651.

(40) Evans, E. Faradav Discuss. 1998, 111, 1.

(41) Butt, H. J.; Jaschke, M. Nanotechnology 1995, 6, 1.

(42) Rief, M.; Gautel, M.; Oesterhelt, F.; Fernandez, J. M.; Gaub, H. E. Science 1997, 276, 1109.

(43) Evans, E.; Ritchie, K. Biophvs. I. 1997, 72, 1541.

(44) Bieri, O.; Wirz, J.; Hellrung, B.; Schutkowski, M.; Drewello, M.; Kiefhaber, T. Proc. Natl. Acad. Sci. U.S.A 1999, 96, 9597.

(45) Krieger, F.; Fierz, B.; Bieri, O.; Drewello, M.; Kiefhaber, T. L. Mol. Biol. 2003, 332, 265.

(46) Gräter, F.; Grubmüller, H. L. Struct. Biol. 2007, 157, 557.

(47) Nunes, J. M.; Hensen, U.; Ge, L.; Lipinsky, M.; Helenius, J.; Grubmüller, H.; Müller, D. I. Anoew. Chem. Int. Ed. 2010, 49, 3528. 